\title{
Caracterização de genótipos de soja na região dos Cerrados quanto à reação à podridão vermelha da raiz, causada pelos fungos $F$ usarium tucumaniae e Fusarium brasiliense
}

\author{
Austeclinio Lopes de Farias Neto ${ }^{1}$, Alexei de Campos Dianese ${ }^{2}$, Mateus Rollemberg Santin ${ }^{3}$, \\ Bárbara Cristina do Couto ${ }^{3}$
}

\section{RESUMO}

A podridão vermelha da raiz de soja vem crescendo em importância, a cada ano, no Brasil, com aumento substancial de participação nas perdas de produtividade. Na região dos Cerrados, são escassos os dados de campo sobre a reação de cultivares à doença. Assim, este trabalho teve como objetivo a caracterização, em campo, de uma série de genótipos de soja, quanto à resistência à podridão vermelha da raiz, em solos naturalmente infestados. Foram testados 71 genótipos de soja, sendo 16 do ciclo de maturação precoce, 28 do ciclo de maturação médio e 27 do ciclo de maturação tardio, em quatro localidades, no entorno do Distrito Federal. O delineamento experimental foi o de blocos ao acaso, com quatro repetições. A caracterização foi realizada por meio dos níveis de incidência e severidade dos sintomas foliares. Genótipos com altos níveis de resistência à doença foram observados nos três grupos de maturação, em cultivares e linhagens em fase final de melhoramento.

Palavras-chave: resistência genética, Glycine max, morte súbita

\section{ABSTRACT}

\section{Reaction of soybean cultivars}

\section{to the sudden death syndrome pathogens Fusarium tucumaniae and F. brasiliense}

Loss due to soybean sudden death syndrome (SDS) has increased considerably in Brazil in recent years. Field data on reaction of soybean genotypes to SDS infection in the Cerrados is almost nonexistent. Therefore, the objective of this work was to characterize the reaction of soybean genotypes, adapted to the Cerrados, to SDS infection on field trials in areas with natural inoculum. Seventy one soybean genotypes were used in the experiments, sixteen early season, twenty eight midseason and twenty seven late season genotypes, in field trials located in four different rural properties around Brazil's Federal District. The experimental design used was the randomized complete block, with four repetitions. The characterization was performed using the levels of disease incidence and severity of foliar symptoms Genotypes with good levels of disease resistance were observed in all three maturity groups, cultivars and lines in the final stages of development.

Key words: genetic resistance, Glycine max, root rot.

\footnotetext{
RRecebido para publicação em 15/12/2011 e aprovado em 19/12/2012.

${ }^{1}$ Engenheiro-Agrônomo, Ph.D. Embrapa Agrossilvipastoril, Avenida das Jacarandás, 2639, Centro, 78550-003, Sinop, Mato Grosso, Brasil. austeclinio.farias@embrapa.br (autor para correspondência)

${ }^{2}$ Biólogo, Doutor. Embrapa Cerrados, Rodovia BR 020, Km 18, 73310-970, Planaltina, Distrito Federal, Brasil. alexei.dianese@cpac.embrapa.br

${ }^{3}$ Engennheiro-Agrônomo. Embrapa Cerrados, Rodovia BR 020, Km 18, 73310-970, Planaltina, Distrito Federal, Brasil. mateusantin@ gmail.com; barbaracristine.couto@wehrmann.com.br
} 


\section{INTRODUÇÃO}

Dentre as várias doenças fúngicas que afetam a cultura da soja [Glycine max (L.) Merr.], no Brasil, a podridão vermelha da raiz (PVR), cujos agentes etiológicos são os fungos Fusarium tucumaniae e F. brasiliense (Aoki et al., 2005; Oliveira, 2010), vem apresentando incrementos elevados de participação nas reduções de produtividade de grãos, encontrando-se disseminada em todas as regiões do país (Embrapa Soja, 2001; Embrapa Soja, 2006). Os patógenos são fungos de solo, que infectam as raízes das plantas hospedeiras. A doença causa redução significativa na nodulação e perda de massa radicular. Os sintomas na parte aérea incluem clorose internerval, necrose foliar e desfolha prematura (Hartman et al., 1997; Farias Neto et al., 2006). Os sintomas foliares desenvolvem-se, com alta taxa de progresso, geralmente durante o estádio reprodutivo da soja (Gibson et al., 1994).

Não existe controle químico adequado para a PVR, sendo que algumas práticas culturais têm sido capazes de reduzir seu impacto (Rupe \& Hartman, 1999). Rupe et al. (1997) demonstraram que a rotação de soja com sorgo e trigo reduziu significativamente a população do patógeno. Solos bem drenados (Silva et al., 2002), juntamente com o uso de cultivares resistentes, têm sido as práticas agronômicas mais eficientes para reduzir o impacto dessa doença (Farias Neto et al., 2006; Embrapa Soja, 2002). No entanto, a resistência à doença é parcial, tendo em vista que mesmo os genótipos considerados resistentes apresentaram sintomas típicos da podridão vermelha da raiz (Yorinori, 2000; Silva et al., 2002; Gásperi et al., 2003).

Na região dos Cerrados, são escassos os dados de campo sobre a reação de cultivares de soja à PVR, de forma que a caracterização de genótipos quanto à resistência a esta doença é de fundamental importância para a região.

Este trabalho teve como objetivo a caracterização de genótipos de soja quanto à resistência à podridão vermelha da raiz, em áreas naturalmente infestadas com os fungos.

\section{MATERIAL E MÉTODOS}

Amostras de plantas com sintomas de PVR foram coletadas, nas áreas experimentais, e o isolamento do organismo foi efetuado a partir de fragmentos da raiz, desinfestados superficialmente em solução de hipoclorito de sódio $(2,5 \%)$. Os fragmentos desinfestados foram, então, transferidos para placas de Petri com meio de cultura (1 litro de BDA + $10 \mathrm{mg}$ estreptomicilina). A seguir, as placas foram incubadas a $25{ }^{\circ} \mathrm{C}$ (fotoperíodo de $12 \mathrm{~h}$ ) e avaliadas diariamente para que a presença de Fusarium spp. fosse detectada.

Após detectadas, as colônias de Fusarium spp. foram transferidas para placas de Petri, contendo o meio de cultura acima descrito, e incubadas a $25^{\circ} \mathrm{C}$ (fotoperíodo de $12 \mathrm{~h}$ ) por sete dias. As placas com os isolados do patógeno eram, então, armazenadas em uma câmara fria, a $10^{\circ} \mathrm{C}$.
Amostras de cada isolado foram analisadas sobre uma lâmina, que recebeu uma gota de água destilada. Foram então medidos os esporos, conforme os critérios morfológicos descritos por Aoki et al. (2005). Foram feitas 50 medições de cada estrutura dos isolados.

Foram caracterizados 71 genótipos de soja, sendo 16 do ciclo de maturação precoce, 28 do ciclo de maturação médio e 27 do ciclo de maturação tardio. Os ensaios foram instalados, separadamente, por grupo de maturação. O delineamento experimental utilizado foi o de blocos ao acaso, com quatro repetições. Cada unidade experimental correspondeu a uma linha de cinco metros de comprimento, plantada com um único genótipo. O espaçamento entre as linhas foi de $50 \mathrm{~cm}$ e a população de plantas em torno de $300 \mathrm{mil} / \mathrm{ha}$, com as sementes sendo incorporadas ao solo na profundidade de $3 \mathrm{~cm}$. O ensaio foi conduzido de 02/11/2008 a 07/11/2008, em quatro propriedades, no Estado de Goiás, Fazendas MR (MR) e Salto Verde (SV) em Luziânia, Pamplona (PA) em Cristalina e Três Pinheiros (TP), em Formosa, em áreas com histórico de ocorrência da podridão vermelha da raiz.

A incidência (DI) e a severidade (DS) da podridão vermelha da raiz foram avaliadas no estádio fenológico R6 (Ferh et al., 1971). A incidência (DI) foi avaliada, em cada parcela, como a percentagem de plantas em relação ao total com sintomas foliares típicos da doença. Já a severidade foliar (DS) foi avaliada, em cada parcela, utilizando-se a seguinte escala diagramática (Farias Neto et al., 2006):

$1=0$ a $10 \%$ da área foliar com clorose ou de 1 a $5 \%$ da área foliar com necrose; $2=10$ a $20 \%$ da área foliar com clorose ou de 6 a $10 \%$ da área foliar com necrose; $3=20$ a $40 \%$ da área foliar com clorose ou de 10 a $20 \%$ da área foliar com necrose; $4=40$ a $60 \%$ da área foliar com clorose ou de 20 a 40\% da área foliar com necrose; $5=>$ que $60 \%$ da área foliar com clorose ou $>40 \%$ da área foliar com necrose; 6 $=$ até $33 \%$ de desfolha; $7=$ de 34 a $66 \%$ de desfolha; $8=>$ que $66 \%$ de desfolha e $9=$ morte prematura da planta.

Um índice da doença (ID) foi calculado, usando-se a fórmula [(DI) x (DS)]/9 (Farias Neto et al., 2006).

Os dados obtidos foram submetidos à análise de normalidade dos erros, pelo teste de Shapiro-Wilk; à análise de variância por grupo de maturação e por local, e à análise conjunta por grupo de maturação, utilizando-se o programa SAS (SAS Institute, 2000). Considerando-se os resultados das análises de variância conjunta, as médias dos genótipos por grupo de maturação e por local, significativas no teste F, foram agrupadas por Scott-Knott. Para isto, foi utilizado o aplicativo computacional Genes (Cruz, 2008).

\section{RESULTADOS E DISCUSSÃO}

Foram observados, nos quatro locais de teste, sintomas típicos da PVR nas raízes e nas folhas das plantas. Isolados retirados de raízes infectadas foram identificados em laboratório, seguindo-se os critérios morfológicos descritos por Aoki et al. (2005), como sendo F. brasiliense 
e F. tucumaniae. Nas duas propriedades em Luziânia, MR e SV, foram identificados isolados apenas de $F$. tucumaniae; já na propriedade TP, em Formosa, somente isolados de $F$. brasiliense e, em Cristalina, na propriedade PA, ocorreram as duas espécies ( $F$. tucumaiae e $F$. brasiliense). As médias de incidência e de severidade dos sintomas da doença foram altas nos locais MR, SV e PA e baixas em TP, e não foram observados sintomas da doença nos ensaios de genótipos de ciclo precoce e médio, no local TP. Nos locais MR, SV e PA, foi possível avaliar inclusive os genótipos precoces, que normalmente escapam à doença, em função do ciclo (Rupe et al., 1995). Os sintomas nas folhas tiveram início, de uma forma geral, no estágio R4/R5. Foi observada variabilidade genética para reação à doença em todos os ensaios.

Segundo o teste de Shapiro-Wilk, os erros estavam normalmente distribuídos. As análises conjuntas realizadas nos três grupos de maturação, precoce (Tabela 1), médio (Tabela 2) e tardio (Tabela 3), mostraram efeitos significativos a $1 \%$ de probabilidade para as fontes de variação "locais", "cultivares" e "locais*cultivares". Por causa da presença de efeitos significativos da interação "locais*cultivares", os genótipos de cada grupo de maturação foram avaliados por local. A presença de interação genótipo*ambiente é comum em ensaios de reação de genótipos à PVR, tendo em vista o controle quantitativo do caráter, com grande influência do ambiente (Igbal et al., 2001; Nijti et al., 2001).

As médias do índice da doença dos ensaios precoces, conduzidos nas fazendas MR, SV e PA, foram de 4,90, 8, 12 e 5,35, respectivamente (Tabela 4), perfazendo média de 6,13 . Nesses ensaios não foi observada diferença significativa entre os genótipos, para reação a PVR no local PA, enquanto, nos locais MR e SV foram observados dois e quatro grupamentos significativamente diferentes, respectivamente. Muitos genótipos apresentaram, nesses ensai-

Tabela 1. Análises de variância conjunta para índice de reação de genótipos de soja de ciclo precoce à PVR (ID), avaliada em três locais no Estado de Goiás, 2009

\begin{tabular}{lrcrc}
\hline $\begin{array}{l}\text { Fonte de } \\
\text { variação }\end{array}$ & GL & QM & Valor F & Pr>F \\
\hline Local & 2 & 570,537197 & 14,72 & $<0,0001$ \\
Cultivar & 15 & 395,073655 & 10,19 & $<0,0001$ \\
Local*Cultivar & 30 & 115,460459 & 2,98 & $<0,0001$ \\
\hline
\end{tabular}

Tabela 2. Análises de variância conjunta para índice de reação de genótipos de soja de ciclo médio à PVR (ID), avaliada em quatro locais no Estado de Goiás, 2009

\begin{tabular}{lrrrr}
\hline $\begin{array}{l}\text { Fonte de } \\
\text { variação }\end{array}$ & GL & QM & Valor F & Pr>F \\
\hline Local & 3 & 2398,78201 & 56,44 & $<0,0001$ \\
Cultivar & 27 & 277,34649 & 6,53 & $<0,0001$ \\
Local*Cultivar & 81 & 120,03282 & 2,82 & $<0,0001$ \\
\hline
\end{tabular}

os, bons níveis de resistência aos patógenos, com destaque para os cultivares BRSMG 751, BRS Raíssa, BRSMG 750S e BRSGO 7560.

Para os ensaios de ciclo de maturação médio, foram observadas médias de ID de 11,18, 14,60, 11,60 e 2,30, nas fazendas MR, SV, PA e TP, respectivamente (Tabela 5). A média geral dos ensaios foi de 10,08. Assim como no ensaio de ciclo precoce, não foram observados sintomas foliares de PVR, na Fazenda TP, no ensaio de ciclo médio. Altos níveis de resistência foliar a PVR foram observados em vários cultivares, com destaque para BRS Milena, BRSMG-810C, BRSMG-850GRR e BRS Valiosa. Esses cultivares apresentam-se como ótimas opções de cultivo para áreas infestadas pelo fungo causador da PVR.

Os ensaios de ciclo tardio apresentaram os maiores valores entre todos os ensaios (Tabela 6), atingindo uma média geral de 14,57. Os maiores índices observados nos

Tabela 3. Análises de variância conjunta para índice de reação de genótipos de soja de ciclo tardio à PVR (ID), avaliada em quatro locais no estado de Goiás, 2009

\begin{tabular}{lrrrr}
\hline $\begin{array}{l}\text { Fonte de } \\
\text { variação }\end{array}$ & GL & QM & Valor F & Pr>F \\
\hline Local & 3 & 1670,98368 & 40,39 & $<0,0001$ \\
Cultivar & 26 & 1297,33329 & 31,36 & $<0,0001$ \\
Local*Cultivar & 78 & 136,14492 & 3,29 & $<0,0001$ \\
\hline
\end{tabular}

Tabela 4. Média geral de índice de reação de genótipos de soja de ciclo de maturação precoce à PVR (ID), avaliada em três locais no Estado de Goiás, 2009

\begin{tabular}{lccr}
\hline \multicolumn{4}{c}{ Índice de PVR (ID) } \\
\hline \multicolumn{4}{c}{ Locais $^{(1)}$} \\
\hline Genótipos & MR & SV & PA \\
\hline BRS ROSA & $10,80 \mathrm{a}$ & $34,10 \mathrm{a}$ & $10,00 \mathrm{a}$ \\
BRS FLORA & $14,00 \mathrm{a}$ & $25,10 \mathrm{~b}$ & $5,64 \mathrm{a}$ \\
TMG 103 & $19,30 \mathrm{a}$ & $10,00 \mathrm{c}$ & $5,00 \mathrm{a}$ \\
MSOY 8000 & $10,30 \mathrm{a}$ & $11,70 \mathrm{c}$ & $11,38 \mathrm{a}$ \\
MSOY 8199 & $7,60 \mathrm{a}$ & $8,60 \mathrm{c}$ & $14,60 \mathrm{a}$ \\
MSOY 8008 & $6,70 \mathrm{a}$ & $9,00 \mathrm{c}$ & $6,24 \mathrm{a}$ \\
BRSGO GOIÂNIA & $1,90 \mathrm{~b}$ & $8,10 \mathrm{c}$ & $5,96 \mathrm{a}$ \\
BRS NINA & $0,90 \mathrm{~b}$ & $4,10 \mathrm{~d}$ & $7,53 \mathrm{a}$ \\
BRS IARA & $4,20 \mathrm{~b}$ & $5,30 \mathrm{~d}$ & $1,90 \mathrm{a}$ \\
BRS FAVORITA & $0,30 \mathrm{~b}$ & $3,60 \mathrm{~d}$ & $6,32 \mathrm{a}$ \\
MSOY 8001 & $2,00 \mathrm{~b}$ & $6,80 \mathrm{c}$ & $2,77 \mathrm{a}$ \\
BRSMG-751 & $0,00 \mathrm{~b}$ & $1,10 \mathrm{~d}$ & $2,96 \mathrm{a}$ \\
BRSGO RAÍSSA & $0,40 \mathrm{~b}$ & $0,40 \mathrm{~d}$ & $2,40 \mathrm{a}$ \\
BRSMG 750S & $0,00 \mathrm{~b}$ & $1,10 \mathrm{~d}$ & $1,94 \mathrm{a}$ \\
BRSGO 7560 & $0,00 \mathrm{~b}$ & $0,07 \mathrm{~d}$ & $1,20 \mathrm{a}$ \\
EMGOPA 316 & $0,20 \mathrm{~b}$ & $1,00 \mathrm{~d}$ & $0,00 \mathrm{a}$ \\
\hline ID médio por local & 4,90 & 8,12 & 5,35 \\
\hline (1)MR = Fazenda Moisés Rapache, Luziânia; SV = Fazenda Salto \\
Verde, Luziânia; PA = Fazenda Pamplona, Cristalina \\
Médias seguidas pela mesma letra na coluna não diferem \\
estatisticamente entre & si pelo teste de Scott-Knott, a 5\% de \\
probabilidade. & & & \\
& &
\end{tabular}


ensaios de ciclo tardio em relação aos de ciclo médio, e destes em relação aos de ciclo precoce, podem ser explicados pela maior exposição dos genótipos de soja de ciclo tardio ao patógeno (Westphal et al., 2008). Quando são testados em casa de vegetação, as médias de forma geral são similares para genótipos de diferentes ciclos de maturação (Farias Neto et al., 2008). Entretanto, os testes de campo, especialmente em fases finais de programas de melhoramento, refletem mais a realidade do que os testes realizados em casa de vegetação (Farias Neto et al., 2008). Os cultivares BRS Tuiuiú e P98R91 apresentaram os maiores índices, respectivamente. Os sintomas foliares menos severos foram observados para os cultivares BRS Serena e BRS Sambaíba, mostrando uma alta resistência à doença.

Reduções severas em produtividade podem ocorrer por causa da PVR, dependendo da intensidade dos sinto- mas (Rupe et al., 1999). O efeito da doença na produtividade depende fundamentalmente do estádio fenológico da planta, da extensão dos sintomas radiculares e dos sintomas foliares e do progresso da doença a partir desses sintomas. Farias Neto et al. (2006), trabalhando com parcelas inoculadas e não inoculadas com Fusarium spp., observaram reduções em produtividade de grãos de até $27 \%$, em cultivares susceptíveis, que apresentaram severos sintomas foliares, correspondendo a cerca de $30 \%$ de desfolha no estádio R6. Dessa forma, Farias Neto et al. (2006) observaram que, para cada unidade de índice de PVR, ocorreu, em média, $1 \%$ de queda em produtividade de grãos (Farias Neto et al., 2006). Com base nos níveis de doença observados nos presentes ensaios, as perdas em produtividade de grãos estimadas para os genótipos susceptíveis poderiam atingir índices muito significativos.

Tabela 5. Média geral de índice de reação de genótipos de soja de ciclo de maturação médio à PVR (ID), avaliada em quatro locais no Estado de Goiás, 2009

\begin{tabular}{|c|c|c|c|c|}
\hline \multicolumn{5}{|c|}{ Índice de PVR (ID) } \\
\hline \multicolumn{5}{|c|}{ Locais $^{(1)}$} \\
\hline Genótipos & MR & SV & PA & TP \\
\hline BR95-3723 & $24,80 \mathrm{a}$ & $29,40 \mathrm{a}$ & $30,90 \mathrm{a}$ & $8,50 \mathrm{a}$ \\
\hline TMG 108 & $26,00 \mathrm{a}$ & $36,10 \mathrm{a}$ & $12,20 \mathrm{~b}$ & $6,20 \mathrm{a}$ \\
\hline TMG 106 & $26,60 \mathrm{a}$ & $21,10 \mathrm{~b}$ & $26,90 \mathrm{a}$ & $5,90 \mathrm{a}$ \\
\hline MSOY-8287 & $13,80 \mathrm{~b}$ & $29,40 \mathrm{a}$ & $25,40 \mathrm{a}$ & $3,00 \mathrm{a}$ \\
\hline ROBR00-121478 (3) & - & $36,90 \mathrm{a}$ & $7,60 \mathrm{~b}$ & $8,10 \mathrm{a}$ \\
\hline P98R62 & $22,30 \mathrm{a}$ & $24,60 \mathrm{~b}$ & $9,80 \mathrm{~b}$ & $3,60 \mathrm{a}$ \\
\hline BRSGO PARAÍSO & $25,30 \mathrm{a}$ & $15,10 \mathrm{c}$ & $10,40 \mathrm{~b}$ & $1,60 \mathrm{a}$ \\
\hline P98R31 & $24,60 \mathrm{a}$ & $16,40 \mathrm{c}$ & $16,30 \mathrm{~b}$ & $3,40 \mathrm{a}$ \\
\hline BRS XINGU & $20,20 \mathrm{a}$ & $15,50 \mathrm{c}$ & $11,90 \mathrm{~b}$ & $1,70 \mathrm{a}$ \\
\hline ROBR00-40220 & $12,40 \mathrm{~b}$ & $32,20 \mathrm{a}$ & $11,30 \mathrm{~b}$ & $2,00 \mathrm{a}$ \\
\hline MSOY 8411 & $12,00 \mathrm{~b}$ & $16,80 \mathrm{c}$ & $6,00 \mathrm{~b}$ & $3,00 \mathrm{a}$ \\
\hline ROBR00-122191 & $14,30 \mathrm{~b}$ & $12,00 \mathrm{c}$ & $8,60 \mathrm{~b}$ & $0,90 \mathrm{a}$ \\
\hline BRSGO CHAPADÕES & $15,20 \mathrm{~b}$ & $5,90 \mathrm{~d}$ & $13,30 \mathrm{~b}$ & $0,10 \mathrm{a}$ \\
\hline BRSGO LUZIÂNIA & $11,90 \mathrm{~b}$ & $11,90 \mathrm{c}$ & $7,40 \mathrm{~b}$ & $0,92 \mathrm{a}$ \\
\hline BRS TUCANO & $9,40 \mathrm{~b}$ & $14,60 \mathrm{c}$ & $3,50 \mathrm{~b}$ & $1,10 \mathrm{a}$ \\
\hline MTBR00-45793 & $4,70 \mathrm{c}$ & $11,10 \mathrm{c}$ & $12,30 \mathrm{~b}$ & $0,53 \mathrm{a}$ \\
\hline BRS CELESTE ${ }^{(2)}$ & $10,10 \mathrm{~b}$ & - & $16,50 \mathrm{~b}$ & $1,70 \mathrm{a}$ \\
\hline EMGOPA-315 & $10,60 \mathrm{~b}$ & $6,70 \mathrm{~d}$ & $7,50 \mathrm{~b}$ & $1,00 \mathrm{a}$ \\
\hline MTBR00-44058 & $3,30 \mathrm{c}$ & $14,30 \mathrm{c}$ & $6,70 \mathrm{~b}$ & $0,70 \mathrm{a}$ \\
\hline BRSMT PINTADO & $3,40 \mathrm{c}$ & $11,10 \mathrm{c}$ & $8,10 \mathrm{~b}$ & $0,40 \mathrm{a}$ \\
\hline BRS CONQUISTA & $6,70 \mathrm{c}$ & $6,70 \mathrm{~d}$ & $7,60 \mathrm{~b}$ & $0,80 \mathrm{a}$ \\
\hline BRAS00-11610 & $3,20 \mathrm{c}$ & $1,30 \mathrm{~d}$ & $14,10 \mathrm{~b}$ & $2,60 \mathrm{a}$ \\
\hline MTBR00-122062 & $0,60 \mathrm{c}$ & $12,10 \mathrm{c}$ & $6,70 \mathrm{~b}$ & $1,00 \mathrm{a}$ \\
\hline BRSGO INDIARA & $2,50 \mathrm{c}$ & $5,20 \mathrm{~d}$ & $10,90 \mathrm{~b}$ & $1,00 \mathrm{a}$ \\
\hline BRS VALIOSA & $0,20 \mathrm{c}$ & $0,30 \mathrm{~d}$ & $15,40 \mathrm{~b}$ & $1,10 \mathrm{a}$ \\
\hline BRSMG $810 \mathrm{C}^{(2)}$ & - & $5,60 \mathrm{~d}$ & $4,60 \mathrm{~b}$ & $0,40 \mathrm{a}$ \\
\hline BRSMG 850GRR & $3,90 \mathrm{c}$ & $1,60 \mathrm{~d}$ & $6,50 \mathrm{~b}$ & $2,70 \mathrm{a}$ \\
\hline BRS MILENA & $0,00 \mathrm{c}$ & $0,10 \mathrm{~d}$ & $6,80 \mathrm{~b}$ & $0,50 \mathrm{a}$ \\
\hline ID médio por local & 11,84 & 14,60 & 11,61 & 2,30 \\
\hline
\end{tabular}

Rev. Ceres, Viçosa, v. 60, n.2, p. 215-220, mar/abr, 2013 
Tabela 6. Média geral de índice de reação de genótipos de soja de ciclo de maturação tardio à PVR (ID), avaliada em quatro locais no Estado de Goiás, 2009

\begin{tabular}{|c|c|c|c|c|}
\hline \multicolumn{5}{|c|}{ Índice de PVR (ID) } \\
\hline \multicolumn{5}{|c|}{ Locais $^{(1)}$} \\
\hline Genótipos & MR & SV & PA & TP \\
\hline BRSMS TUIUIU & $48,88 \mathrm{a}$ & $54,81 \mathrm{a}$ & 29,18 a & $41,29 \mathrm{a}$ \\
\hline P98R91 & $51,11 \mathrm{a}$ & $49,33 \mathrm{a}$ & $25,81 \mathrm{a}$ & $25,33 \mathrm{~b}$ \\
\hline BRS TIANA & $43,70 \mathrm{a}$ & $44,44 \mathrm{a}$ & $14,72 \mathrm{~b}$ & $22,22 \mathrm{~b}$ \\
\hline BRSMT UIRAPURU & $36,96 \mathrm{~b}$ & $35,00 \mathrm{~b}$ & $18,55 \mathrm{~b}$ & $12,47 \mathrm{c}$ \\
\hline BRS AMARALINA & $36,96 \mathrm{~b}$ & $35,00 \mathrm{~b}$ & $5,16 \mathrm{c}$ & $15,64 \mathrm{c}$ \\
\hline TMG 113 & $23,66 \mathrm{c}$ & $26,51 \mathrm{c}$ & $20,00 \mathrm{~b}$ & $8,33 \mathrm{c}$ \\
\hline TMG 117 & $21,14 \mathrm{c}$ & $22,22 \mathrm{c}$ & $18,51 \mathrm{~b}$ & $7,29 \mathrm{c}$ \\
\hline MSOY 8925 & $21,85 \mathrm{c}$ & $23,14 \mathrm{c}$ & $18,44 \mathrm{~b}$ & $4,77 \mathrm{~d}$ \\
\hline TMG 121 & $21,51 \mathrm{c}$ & $25,14 \mathrm{c}$ & $13,33 \mathrm{~b}$ & $7,88 \mathrm{c}$ \\
\hline P99R01 & $20,55 \mathrm{c}$ & $26,29 \mathrm{c}$ & $11,48 \mathrm{~b}$ & $6,75 \mathrm{c}$ \\
\hline BRS CELESTE & $19,55 \mathrm{c}$ & $17,03 \mathrm{c}$ & $19,07 \mathrm{~b}$ & $7,40 \mathrm{c}$ \\
\hline BRS GRALHA & $13,33 \mathrm{~d}$ & $15,55 \mathrm{c}$ & $16,96 \mathrm{~b}$ & $9,63 \mathrm{c}$ \\
\hline BR-IAC 21 & $20,92 \mathrm{c}$ & $9,44 \mathrm{~d}$ & $12,22 \mathrm{~b}$ & $5,87 \mathrm{~d}$ \\
\hline MSOY 8800 & $15,03 \mathrm{c}$ & $21,33 \mathrm{c}$ & $4,44 \mathrm{c}$ & $4,11 \mathrm{~d}$ \\
\hline TMG115 & $8,70 \mathrm{~d}$ & $13,51 \mathrm{~d}$ & $16,29 \mathrm{~b}$ & $1,87 \mathrm{~d}$ \\
\hline BRS PÉTALA & $7,92 \mathrm{~d}$ & $17,77 \mathrm{c}$ & $10,55 \mathrm{c}$ & $4,11 \mathrm{~d}$ \\
\hline BRS RAIMUNDA & $11,85 \mathrm{~d}$ & $10,00 \mathrm{~d}$ & $13,59 \mathrm{~b}$ & $2,55 \mathrm{~d}$ \\
\hline BRS GISELE & $5,83 \mathrm{~d}$ & $11,92 \mathrm{~d}$ & $16,85 \mathrm{~b}$ & $2,03 \mathrm{~d}$ \\
\hline BRS BALISA RR & $9,16 \mathrm{~d}$ & $20,66 \mathrm{c}$ & $2,22 \mathrm{c}$ & $3,40 \mathrm{~d}$ \\
\hline BRS JIRIPOCA & $7,29 \mathrm{~d}$ & $18,51 \mathrm{c}$ & $5,55 \mathrm{c}$ & $2,88 \mathrm{~d}$ \\
\hline BRS GRACIOSA RR & $2,77 \mathrm{~d}$ & $6,66 \mathrm{~d}$ & $1,85 \mathrm{c}$ & $12,05 \mathrm{c}$ \\
\hline BRS PRINCESA & $4,07 \mathrm{~d}$ & $9,44 \mathrm{~d}$ & $5,42 \mathrm{c}$ & $4,16 \mathrm{~d}$ \\
\hline BRS JULIANA & $3,51 \mathrm{~d}$ & $7,85 \mathrm{~d}$ & $8,66 \mathrm{c}$ & $0,55 \mathrm{~d}$ \\
\hline BRS SILVÂNIA & $3,33 \mathrm{~d}$ & $11,01 \mathrm{~d}$ & $5,74 \mathrm{c}$ & $0,50 \mathrm{~d}$ \\
\hline $\mathrm{CD}-219^{(3)}$ & $12,96 \mathrm{~d}$ & $1,94 \mathrm{~d}$ & - & $0,33 \mathrm{~d}$ \\
\hline BRS SAMBAÍBA & $6,11 \mathrm{~d}$ & $3,33 \mathrm{~d}$ & $0,88 \mathrm{c}$ & $3,64 \mathrm{~d}$ \\
\hline BRS SERENA & $2,83 \mathrm{~d}$ & $6,29 \mathrm{~d}$ & $1,66 \mathrm{c}$ & $2,57 \mathrm{~d}$ \\
\hline ID médio por local & 17,83 & 20,15 & 12,20 & 8,13 \\
\hline
\end{tabular}

${ }^{(1)}$ MR = Fazenda Moisés Rapachi, Luziânia; SV = Fazenda Salto Verde, Luziânia; PA = Fazenda Pamplona, Cristalina; TP = Fazenda Três Pinheiros, Formosa. ${ }^{(2)}$ Média do índice de doença (ID; 0-100) para cada genótipo nas quatro localidades. ${ }^{(3)}$ Genótipo avaliado em três das quatro localidades.

\section{CONCLUSÕES}

Foi observada alta variabilidade genética, entre os genótipos avaliados, para resistência à podridão vermelha da raiz.

Fontes de resistência à doença foram observadas, nos três grupos de maturação, em cultivares comerciais e em linhagens em fase final de melhoramento.

Os genótipos de ciclo de maturação precoce avaliados apresentaram índices de PVR inferiores aos dos genótipos de ciclo de maturação médio e tardio.

\section{AGRADECIMENTO}

Os autores agradecem ao pesquisador Fávio Dessaune Tardin, pelas contribuições nas análises estatísticas dos dados.

\section{REFERÊNCIAS}

Aoki T, O'donnell K, Homma Y \& Lattanzi AR (2005) Sudden death syndrome of soybean in South America is caused by four species of Fusarium: Fusarium brasiliense sp. nov., F. Cuneirostrum sp. nov., F. Tucumaniae, and F. virguliforme. Mycoscience, 6:162-183.

Cruz CD (2008) Programa genes: versão Windows; aplicativo computacional em genética e estatística. Viçosa, UFV. 648p.

Embrapa Soja (2001) Tecnologias de produção de soja - região central do Brasil - 2001/2002. Londrina, Embrapa Soja. 267p. (Documentos, 167).

Embrapa Soja (2002) Tecnologias de produção de soja - região central do Brasil - 2003. Londrina, Embrapa Soja/Embrapa Cerrados/Embrapa Agropecuária Oeste/ ESALQ. 199p. (Sistemas de produção, 1).

Embrapa Soja (2006) Tecnologias de produção de soja - região central do Brasil - 2007 Londrina, Embrapa Soja/Embrapa Cerrados/Embrapa Agropecuária Oeste/ ESALQ. 199p. (Sistemas de produção, 11). 
Farias Neto AL, Hartman GL, Pedersen WL, Li S, Bollero GA \& Diers BW (2006) Irrigation and inoculation treatments that increase the severity of soybean sudden death syndrome in the field. Crop Science, 46:2547-2554.

Farias Neto AL, Schimidt M, Hartman GL, Li S \& Diers BW (2008) Inoculation methods under greenhouse conditions for evaluating soybean resistance to sudden death syndrome. Pesquisa Agropecuária Brasileira, 43:1475-1482.

Ferh WR, Caviness CE, Burmood DT, Pennington JS (1971) Stage of development descriptions for soybeans, Glycine max (L.) Merrill. Crop Science, 11:929-931.

Gásperi AC, Prestes AM, Costamilan LM (2003) Reação de cultivares de soja à podridão vermelha da raiz causada por Fusarium solani f. sp. glicynes. Fitopatologia Brasileira, 28:544-547.

Gibson PT, Shenaut MA, Nijti VN, Suttner RJ \& Myers Jr O (1994) Soybean varietal response to sudden death syndrome. In: 24 ${ }^{\text {a }}$ Soybean Seed Research Conference, Chicago. Procedures, American Seed Association. p.20-40.

Hartman GL, Huang YH, Nelson RL \& Noel GR (1997) Germplasm evaluation of Glycine max for resistance to Fusarium solani, the causal organism of sudden death syndrome. Plant Disease, $81: 515-518$.

Igbal MJ, Meksem K, Njiti VN, Kassem MA \& Lightfoot DA (2001) Microsatellite markers identify three additional quantitative trait loci for resistance to soybean sudden-death syndrome (SDS) in Essex x Forrest RILs. Theoretical and Applied Genetics 102:187-192.

Nijti, VN, Johnson JE, Torto TA, Gray LE \& Lightfoot DA (2001) Inoculum rates influences selection for field resistance to soybean sudden death syndrome in the greenhouse. Crop Science, 41:1-6.
Oliveira PRPM (2010) Variabilidade de isolados de Fusarium spp. causadores da podridão vermelha da raiz em soja. Dissertação de Mestrado. Universidade de Brasília, Brasília. 58p.

Rupe JC \& Gbur Junior EE (1995) Effects of plant age, maturity group, and the environment on disease progress of sudden death syndrome of soybean. Plant Disease, 79:139-143.

Rupe JC, Robbins RT \& Gbur Jr EE (1997) Effect of crop rotation on soil population densities of Fusarium solani and Heterodera glycines and on the development of sudden death syndrome of soybean. Crop Protection, 16:575-580.

Rupe JC \& Hartman GL (1999) Sudden death syndrome. In: Hartman GL, Sinclair JB \& Rupe JC (Eds) Compendium of soybean diseases. St. Paul, APS Press. p.37-39.

SAS Institute Inc. (2000). Statistical Analysis System user's guide. 5th ed. Cary, Statistical Analysis System Institute.

Silva JFV, Carneiro GES, Yorinori JT, Almeida AMR, Arias CAA, Kiihl RAS, Almeida LA, Oliveira E, Lima CG, Schober IC, Goulart Filho G, Aliglieri GMG; Gomes JI; Souza NV \& Benato LC (2002) Contribuição ao desenvolvimento de linhagens de soja com resistência a patógenos. Londrina, Embrapa Soja. 43p. (Boletim de pesquisa e desenvolvimento, 1).

Westphal A, Abney TS, Xing LJ \& Shaner GE (2008) Sudden Death Syndrome of Soybean. Disponível em: <http:// www.apsnet.org/education/lessonsplantpath/SuddenDeathSyn>. Acessado em: 24 de junho de 2010.

Yorinori JT (2000) Evolução da ocorrência e da severidade da podridão vermelha da raiz da soja (PVR/SDS) e reação das cultivares comerciais à doença. In: 22 ${ }^{\text {a }}$ Reunião de Pesquisa de Soja da Região Central do Brasil, Cuiabá. Resumos, Embrapa Soja. p.94. 